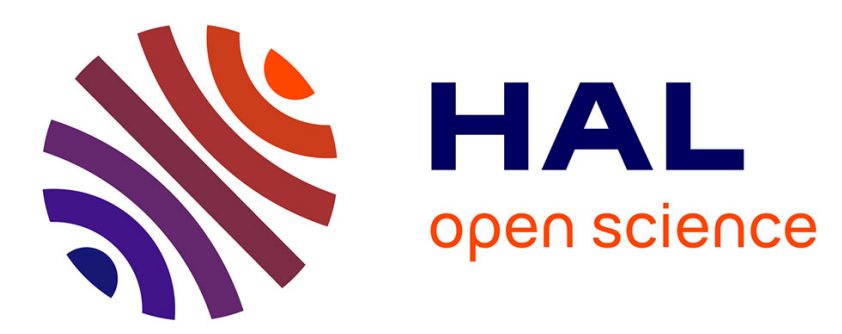

\title{
Le calcul des courants de Foucault en dimension 3, avec le champ électrique comme inconnue. I : Principes
}

\author{
A. Bossavit
}

\section{To cite this version:}

A. Bossavit. Le calcul des courants de Foucault en dimension 3, avec le champ électrique comme inconnue. I: Principes. Revue de Physique Appliquée, 1990, 25 (2), pp.189-197. 10.1051/rphysap:01990002502018900 . jpa-00246178

\section{HAL Id: jpa-00246178 https://hal.science/jpa-00246178}

Submitted on 1 Jan 1990

HAL is a multi-disciplinary open access archive for the deposit and dissemination of scientific research documents, whether they are published or not. The documents may come from teaching and research institutions in France or abroad, or from public or private research centers.
L'archive ouverte pluridisciplinaire HAL, est destinée au dépôt et à la diffusion de documents scientifiques de niveau recherche, publiés ou non, émanant des établissements d'enseignement et de recherche français ou étrangers, des laboratoires publics ou privés. 


\title{
Le calcul des courants de Foucault en dimension 3, avec le champ électrique comme inconnue. I : Principes
}

\author{
A. Bossavit \\ Electricité de France, 1 Avenue du Général de Gaulle, 92141 Clamart Cedex, France
}

(Reçu le 13 juillet 1989, accepté le 7 novembre 1989)

\begin{abstract}
Résumé. - Principes d'une méthode numérique de calcul des courants de Foucault dans des situations tridimensionnelles de géométrie quelconque. L'inconnue est le champ électrique. L'avantage de cette nouvelle méthode par rapport à celles déjà connues qui se servent du champ magnétique est de donner le champ électrique hors des conducteurs, là où il est recherché dans bon nombre de cas.

Abstract. - Principles of a numerical method by which eddy-currents can be computed in three-dimensional situations of general geometry. The primary unknown is the electric field. The comparative advantage of this new method over older ones which take the magnetic field as primary unknown is that it yields the electric field outside conductors, where some specific applications call for its determination.
\end{abstract}

\section{Introduction.}

On sait calculer les courants de Foucault liés à une source de courant variable, en dimension 3, par éléments finis, pour des géométries quelconques, par une méthode maintenant bien établie $[3,6]$, dont le principe consiste à prendre pour inconnues les circulations du champ magnétique le long des arêtes d'un maillage tétraédrique, et éventuellement (là où le courant est nul) les valeurs du potentiel magnétique sur certains sommets du maillage. On s'y référera sous le nom de «méthode $h$-phi».

La méthode $h$-phi ne donne pas accès directement au champ magnétique à l'extérieur des conducteurs. Or dans beaucoup de situations on s'intéresse précisément à ce champ. Par exemple, il est notoire que des arcs électriques intempestifs peuvent apparaître dans certains dispositifs de chauffage par induction. $\mathrm{Si}$ le propos de la modélisation est d'étudier ce phénomène, on aura à calculer le champ électrique dans l'entrefer, plus précisément le champ normal, dans l'air, sur l'interface air-conducteur. Une méthode donnant directement $e$ serait a priori préférable à la méthode $h$-phi pour une telle modélisation.

Or, grâce à une certaine dualité présente dans la structure des équations de Maxwell, il existe une telle méthode, duale de $h$-phi [2]. C'est la « méthode en $e$ », qu'on va présenter ici.
Il existe d'autres méthodes d'éléments finis pour le calcul des courants de Foucault («T-omega » [5], «a-phi» [7], et leurs variantes [9]). Pas plus que $h$ phi elles ne donnent le champ électrique hors des conducteurs. La discussion de leurs rapports entre elles et avec $h$-phi est hors de notre propos (mais voir sur ce point [8]).

Cet article étant consacré aux principes de la méthode en champ électrique (ou « méthode en $e$ »), on discutera un problème modèle, délibérément simplifié, quitte à revenir dans un article ultérieur sur certains problèmes de modélisation propres à la méthode en $e$. Soit donc un conducteur C (Fig. 1),

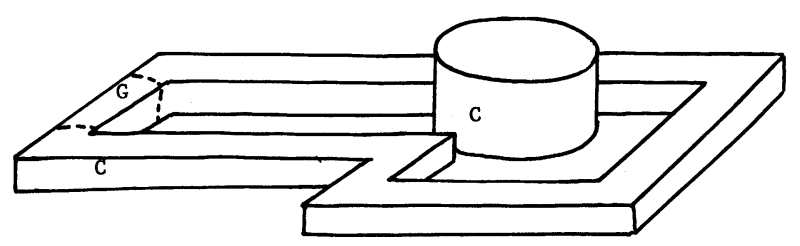

Fig. 1. - La situation étudiée. Dans une partie du domaine conducteur $\mathrm{C}$, soit $\mathrm{G}$, le courant est imposé (indépendamment du champ électrique qui peut exister dans $G$ ).

[The situation under consideration. In part $G$ of the conductive body $\mathrm{C}$, the current density is imposed (irrespective of the electric field in G).] 
éventuellement formé de plusieurs composantes connexes distinctes, et $\mathrm{G}$ une partie de $\mathrm{C}$ (la région "génératrice ») où la densité de courant, soit $j^{\mathrm{g}}$, variable avec le temps, est considérée comme connue. On veut calculer le champ électromagnétique, et tout particulièrement le champ électrique au voisinage des conducteurs.

On ne postule aucune relation entre le courant et le champ électrique dans G. En particulier la loi d'Ohm n'est pas censée s'appliquer dans G. On peut imaginer, pour fixer les idées, un dispositif idéal par lequel des charges électriques seraient transportées à travers $\mathrm{G}$, à contre-courant du champ électrique, le travail dépensé pour ce faire correspondant précisément à l'énergie fournie par le générateur. La structure réelle de celui-ci n'est pas prise en compte.

Ceci ne doit pas être compris comme une limitation de la méthode, mais au contraire comme un élément de souplesse dans la modélisation: car effectivement, dans tous les problèmes concrets, on souhaite pouvoir ignorer les détails de la structure du générateur, tout en tenant compte bien entendu de sa présence. En contrepartie, nous aurons à nous interroger sur la validité de cette "impasse".

Le plan est en deux parties : Formulation (équation du modèle, étude mathématique du modèle, intervention d'une méthode intégrale), puis Discrétisation (éléments finis, étude du modèle discret, approximation de l'opérateur de bord).

Les «Remarques», ainsi que la section 1.2, peuvent être sautées sans inconvénient. La notation " $x \rightarrow f(x)$ " est utilisée pour « la fonction $f$ qui, à $x$, associe la valeur $f(x) »$. Le prime $\left(\psi^{\prime}, e^{\prime}\right.$, $j^{\prime}$, etc.) ne désigne pas des dérivées (la dérivation est toujours notée par un $\partial$ ) mais des fonctions-test ou champs-test. Les barres $(\bar{e}, \bar{\psi}$, etc.) sont réservées aux vecteurs à $n$ composantes (celles-ci étant des degrés de liberté) et aux matrices.

Je remercie F. Rioux-Damidau et P. Chaussecourte de leurs critiques des versions préliminaires, et l'équipe de A. Razek, au LGEP, pour de nombreuses discussions sur leur travail d'implémentation de la méthode en $e$.

\section{Formulation en champ électrique.}

1.1 LeS ÉQuATIONS DE LA MÉTHOdE EN $e$. Soient $b, h, e, j$ les champs de vecteurs familiers : induction, champ magnétique, champ électrique, densité de courant. Ils vérifient les équations de Maxwell dans l'approximation des basses fréquences, soit

$$
\begin{gathered}
\partial_{t} b+\operatorname{rot} e=0 \text { dans } \mathrm{E}, \\
\operatorname{rot} h=j \text { dans } \mathrm{E}
\end{gathered}
$$

(où E désigne l'espace physique, à trois dimensions), la relation

$$
b=\mu h \text { dans } \mathrm{E} \text {, }
$$

et la loi d'Ohm $(j=\sigma e)$ dans $\mathrm{C}-\mathrm{G}$. Si nous convenons que $\sigma=0$ hors de $\mathrm{C}$ et dans $\mathrm{G}$, alors la relation

$$
j=j^{\mathrm{g}}+\sigma e
$$

exprime d'un coup et la loi d'Ohm dans les conducteurs et le fait que le courant est donné (égal à $j^{\mathfrak{g})}$ dans le générateur. La perméabilité $\mu$ dans (3) peut dépendre de la position.

Le point de départ, pour la méthode en $e$, est une «formulation faible » de l'équation (2), comme suit. Nous noterons $\mathbb{E}$ l'espace vectoriel formé par tous les champs de vecteurs de carré intégrable sur tout l'espace et dont le rotationnel est de carré intégrable. Alors, si (2) a lieu, on a aussi, intégrant sur tout l'espace,

$$
\int h \cdot \operatorname{rot} e^{\prime}=\int j \cdot e^{\prime}
$$

pour tout champ de vecteurs $e^{\prime}$ appartenant à $\mathbb{E}$. En effet, une intégration par parties dans le premier membre de (5) donne bien (2). Le champ $e^{\prime}$ n'est pas une entité physique. C'est un champ de vecteurs quelconque, indépendant du temps (le prime n'a rien à voir avec une dérivation) qu'on appelle « champ-test »: si (2) a lieu, (5) a lieu pour $e^{\prime}$ donné, donc, raisonnant à l'envers, (5) « teste » la validité de (2) par rapport au champ $e^{\prime}$. Demander que $h$ et $j$ "passent le test " pour tous les champs $e^{\prime}$ est donc une autre façon d'exprimer l'égalité (2). On l'appelle «formulation faible » de (2).

Remarque 1. Par convention, toute intégrale porte sur l'espace entier, comme dans (5), si le domaine d'intégration n'est pas spécifié.

De (5) on déduit, grâce à (4),

$$
\int\left(\sigma e+j^{\mathrm{g}}\right) \cdot e^{\prime}=\int h \cdot \operatorname{rot} e^{\prime} \quad \forall e^{\prime} \in \mathbb{E} .
$$

Tirons $h$ de (1) et (3) pour le porter dans (6), en dérivant (6), au préalable, par rapport à $t$. Il vient

$$
\begin{aligned}
& \int \partial_{t}\left(\sigma e+j^{\mathrm{g}}\right) \cdot e^{\prime}+\int \frac{1}{\mu} \operatorname{rot} e \cdot \operatorname{rot} e^{\prime}=0 \\
& \forall e^{\prime} \in \mathbb{E},
\end{aligned}
$$

donc si $e$ vérifie les équations (1-4), il doit vérifier (7) à chaque instant de son évolution.

Supposons maintenant $e$ donné à l'instant zéro. On connaîtra donc son évolution en résolvant le problème suivant :

\section{«trouver e dans $\mathbb{E}$ tel que (7) ait lieu »}

(sous-entendu, pour $t \geqslant 0$, éventuellement sur un intervalle de temps $[0, T]$, avec $T$ fini).

Toutefois, un problème de non-unicité apparaît. 
Appelons $\Psi$ l'espace de fonctions (continues et différentiables par morceaux) suivant :

$$
\Psi=\left\{\psi: \psi=c_{i} \text { sur } C_{i}, \quad i=1, \ldots, m\right\}
$$

où les $\mathrm{C}_{i}$ sont les composantes connexes de $\mathrm{C}-\mathrm{G}$, en nombre $m(m=2$ dans le cas de la Fig. 1), et les $c_{i}$ des constantes réelles (non fixées d'avance). Alors si $e$ est solution de (7), $e+\operatorname{grad} \psi$ (où $\psi$ évolue dans $\Psi)$ l'est aussi : donc (7) ne détermine le champ électrique qu'à un gradient près.

Du point de vue physique, c'est naturel, puisque rien n'a été dit sur la présence de charges électriques. Rappelons que la densité de charge est donnée par

$$
\rho=\operatorname{div}(\varepsilon e)
$$

(où $\varepsilon$ est la constante diélectrique du vide). Si rot $e$ est connu, donc $b$, donc $h$, donc $j, e$ est connu là où la loi d'Ohm s'applique, c'est-à-dire là où $\sigma>0$, et on peut (si on y tient) calculer la charge grâce à (9). (Elle n'est non nulle que si $\sigma$ varie spatialement, puisque $\operatorname{div} j=0$.) Mais là où $\sigma=0$, (9) devient au contraire une équation, nécessaire pour compléter la détermination de $e$. La charge $\rho$ hors des conducteurs apparaît comme une autre source du champ, qui superpose ses effets à ceux du courant venu du générateur.

En l'absence de charges d'espace dans la situation étudiée, nous ajoutons donc l'équation

$$
\operatorname{div} e=0 \text { dans } \mathrm{E}-(\mathrm{C}-\mathrm{G})
$$

écrite sous la forme «faible » suivante :

$$
\int e \cdot \operatorname{grad} \psi^{\prime}=0 \quad \forall \psi^{\prime} \in \Psi
$$

(à tout instant $t \geqslant 0$ ). Cette forme faible est en fait plus riche d'information : en plus de $\operatorname{div} e=0$ hors de $C$, elle implique que la charge totale sur chaque $\mathrm{C}_{i}$ est nulle.

Les équations (7) et (10) constituent le modèle mathématique à traiter.

Remarque 2. La charge est nulle hors de $\mathrm{C}$, et aussi dans $\mathrm{C}$ lorsque $\sigma$ est constant (car $\operatorname{div} j=0$, donc $\operatorname{div} \sigma e=\sigma \operatorname{div} e=0$ ). Mais il y a d'autres charges : celles qui apparaissent à la surface de $\mathrm{C}$. En effet, la composante normale de $\varepsilon e$, qui est nulle du côté de l'intérieur d'un conducteur, tout comme celle de $j$, n'a pas de raison d'être nulle du côté de l'extérieur. La différence, ou « saut de la composante normale ", est la charge de surface. On l'obtient donc après avoir calculé $e$.

Remarque 3. A un instant donné, on peut remplacer $e^{\prime}$ dans (5) par $e(t)$, puisque $e^{\prime}$ est quelconque. On obtient alors, en tenant compte de (1),

$$
\int\left(\sigma e+j^{\mathrm{g}}\right) \cdot e+\int h \cdot \partial_{t} b=0
$$

donc, grâce à (3),

$$
\frac{1}{2} \mathrm{~d}_{t} \int \mu|h|^{2}+\int_{\mathrm{C}} \sigma|e|^{2}=-\int_{\mathrm{G}} j^{\mathrm{g}} \cdot e
$$

ce qui est un bilan énergétique facile à interpréter : le second membre est la puissance apportée au système, le premier membre comporte la dérivée par rapport au temps de l'énergie magnétique et la puissance dissipée par effet Joule.

\subsection{LA COHÉRENCE MATHÉMATIQUE DU MODÈLE.} - Bien que les questions mathématiques posées par le modèle (7) (10) (existence, unicité, régularité de la solution, etc.) ne soient pas l'objet de cet exposé, indiquons brièvement comment on peut les traiter en nous limitant toutefois au cas où $\mu=\mu_{0}$ partout. (On peut sauter cette section sans inconvénient.) On s'intéresse d'abord à l'opérateur $K$, de $\mathbb{L}^{2}(\mathrm{C})$ dans $\mathbb{E}$, défini par

$$
K u=x \rightarrow \frac{1}{4 \pi} \int_{\mathrm{C}} \frac{\sigma(y) u(y)}{|x-y|} \mathrm{d} y .
$$

En prenant la restriction de $K u$ à $\mathrm{C}$, on obtient un opérateur compact, auto-adjoint positif, de $\mathbb{L}^{2}(\mathrm{C})$ dans lui-même. Ceci, grâce à la théorie de Fredholm, permet de prouver qu'il existe une infinité dénombrable de couples $\left\{e_{k}, \lambda_{k}\right\}$ (dits « modes propres»), avec $e_{k} \in \mathbb{E}, \lambda_{k}>0$ (les $\lambda_{k}$ sans point d'accumulation), tels que

$$
\int \frac{1}{\mu} \operatorname{rot} e_{k} \cdot \operatorname{rot} e^{\prime}=\lambda_{k} \int \sigma e_{k} \cdot e^{\prime} \quad \forall e^{\prime} \in \mathbb{E}
$$

(poser $e=\mu K u$ pour le voir, en notant que rot rot $K u=\sigma u$.) On cherche alors $e$, solution de (7) (10) sous la forme

$$
e=t \rightarrow \sum_{k} \alpha_{k}(t) e_{k}
$$

pour $t \geqslant 0$, et on constate que chaque $\alpha_{k}$ vérifie une équation différentielle du type

$$
\lambda_{k} \partial_{t} \alpha_{k}+\alpha_{k}=\cdots
$$

où les seconds membres caractérisent $j^{g}$.

(La seule difficulté, une fois intégrées les équations (13), consiste à montrer que $e$ donnée par (12) est bien dans $\mathbb{E}$. Pour cela, il faut que la suite $\sqrt{\lambda_{k}} \alpha_{k}(k=1,2, \ldots)$ soit de carré sommable. Cela dépend de $j^{\mathrm{g}}$, et est vrai sous des hypothèses raisonnables.)

On remarquera que (11) admet des solutions non nulles pour $\lambda_{k}=0$ : ce sont tous les gradients de fonctions appartenant à $\Psi$. Ces solutions, comme on pourrait le montrer, sont nécessairement à divergence non nulle là où $\sigma=0$. En ne les prenant pas en compte dans (12), on vérifie donc (10) implicitement. 
1.3 INTERVENTION D'UN OPÉRATEUR DE BORD. Comment résoudre (7) (10) ? Puisque les intégrales portent sur tout l'espace, on ne peut appliquer directement la méthode des éléments finis. Il faut une certaine préparation, ramenant le problème à un domaine borné, comme suit.

Soit $\mathrm{D}$ un domaine borné (Fig. 2), contenant toute la région intéressante, avec pour frontière une surface S. Nous pouvons supposer que D contient tous les corps ferromagnétiques, s'il s'en trouve, de sorte que $\mu=\mu_{0}$ hors de $\mathrm{D}$.

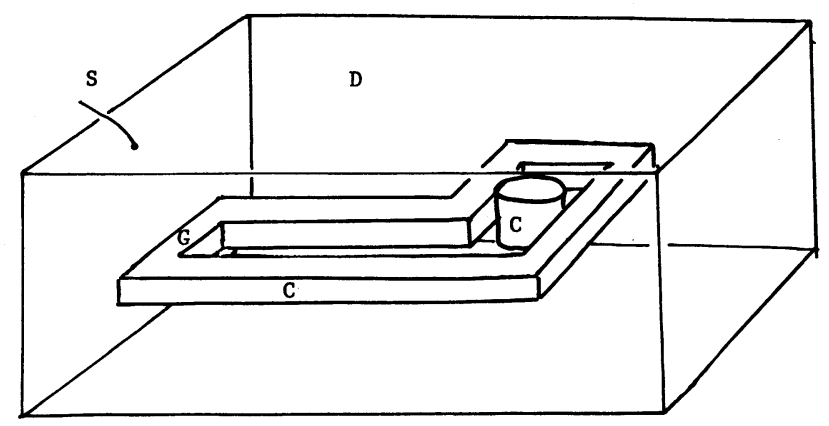

Fig. 2.- Inclusion de $\mathrm{C}$ et $\mathrm{G}$ dans le « domaine de calcul » $\mathrm{D}$, de surface $\mathrm{S}$.

[Inclusion of $\mathrm{C}$ and $\mathrm{G}$ in a « computation domain » $\mathrm{D}$, whose boundary is S.]

Reprenons (7) en l'écrivant sous la forme

$$
\begin{aligned}
& \int_{\mathrm{D}} \partial_{t}\left(\sigma e+j^{\mathrm{g}}\right) \cdot e^{\prime}+\int_{\mathrm{D}} \frac{1}{\mu} \operatorname{rot} e \cdot \operatorname{rot} e^{\prime}+ \\
& \quad+\int_{\mathrm{E}-\mathrm{D}} \frac{1}{\mu_{0}} \operatorname{rot} e \cdot \operatorname{rot} e^{\prime}=0 \quad \forall e^{\prime} \in \mathbb{E} .
\end{aligned}
$$

On va écrire le troisième terme sous la forme d'une intégrale portée par S. D'après une formule d'analyse vectorielle, on a

$$
\begin{aligned}
& \int_{\mathrm{E}-\mathrm{D}} \frac{1}{\mu_{0}} \operatorname{rot} e \cdot \operatorname{rot} e^{\prime}= \\
& \quad=\int_{\mathrm{E}-\mathrm{D}} \operatorname{rot}\left(\frac{1}{\mu_{0}} \operatorname{rot} e\right) \cdot e^{\prime}+\int_{\mathrm{S}}\left(n, \frac{1}{\mu_{0}} \operatorname{rot} e, e^{\prime}\right)
\end{aligned}
$$

où $n$ est la normale à $\mathrm{S}$ sortant de $\mathrm{D}$, et où $(,, \quad)$ désigne le produit mixte.

Or le premier terme à droite de (15) est nul, lorsque $e$ est solution de (14) : en effet, rot $e / \mu_{0}$ est $-\partial_{t} h$, dont le rotationnel est nul puisqu'il n'y a pas de courants dans la région $\mathrm{E}-\mathrm{D}$ (et c'est en fonction de cela que l'on a choisi la frontière $S$ ). Donc, si $e$ est bien solution, le troisième terme de (14) se réduit à une intégrale de surface, que l'on saura traiter numériquement.
En conséquence, introduisons l'outil suivant. Soit $u_{\mathrm{S}}$ un champ de vecteurs superficiel sur $\mathrm{S}$. Soit $u$ le champ de vecteurs défini dans $E-D$, vérifiant

$$
\text { rot } \operatorname{rot} u=0, \operatorname{div} u=0 \text { dans } \mathrm{E}-\mathrm{D},
$$

et de partie tangentielle égale à $u_{\mathrm{s}}$ sur $\mathrm{S}$. On pose alors

$$
Z u_{\mathrm{S}}=n \wedge \operatorname{rot} u
$$

On a ainsi défini un opérateur, qui à $u_{\mathrm{S}}$ associe un autre champ superficiel sur S. Or si $e$ vérifie (7) (10), on a, par définition même de $Z$,

$$
\int_{\mathrm{E}-\mathrm{D}} \frac{1}{\mu_{0}} \operatorname{rot} e \cdot \operatorname{rot} e^{\prime}=\frac{1}{\mu_{0}} \int_{\mathrm{S}} Z e_{\mathrm{S}} \cdot e_{\mathrm{S}}^{\prime}
$$

à tout instant $t \geqslant 0$.

Soit maintenant $\mathbb{E}(D)$ l'espace obtenu en prenant les restrictions à $\mathrm{D}$ des champs appartenant à $\mathbb{E}$, et .

$$
\Psi^{0}(\mathrm{D})=\{\psi \in \Psi: \psi=0 \text { hors de } \mathrm{D}\}
$$

A (7) (10) nous pouvons substituer le modèle suivant :

$$
\begin{aligned}
& \text { Trouver } e \in \mathbb{E}(\mathrm{D}) \text { tel que } \\
& \left.\begin{array}{r}
\int_{\mathrm{D}} \partial_{t}\left(\sigma e+j^{\mathrm{g}}\right) \cdot e^{\prime}+\int_{\mathrm{D}} \frac{1}{\mu} \operatorname{rot} e \cdot \operatorname{rot} e^{\prime}+ \\
+\frac{1}{\mu_{0}} \int_{\mathrm{S}} Z e_{S} \cdot e_{\mathrm{S}}^{\prime}=0 \quad \forall e^{\prime} \in \mathbb{E}(\mathrm{D}), \\
\int_{\mathrm{D}} e \cdot \operatorname{grad} \psi^{\prime}=0 \quad \forall \psi^{\prime} \in \Psi^{0}(\mathrm{D}) .
\end{array}\right\}
\end{aligned}
$$

En effet, si $e$ vérifie (20), son prolongement à tout l'espace défini par (16) est bien solution de (7) (10). Donc (20) donne le champ électrique dans la région D.

Le nouveau modèle (20) a sur (7) (10) l'avantage que l'on recherchait : c'est une formulation variationnelle sur un domaine borné, donc on peut lui appliquer la méthode de Galerkine. La seule difficulté prévisible est le calcul d'une approximation de l'opérateur $Z$.

Donc nous allons procéder ainsi : appliquer la méthode de Galerkine à (18), en supposant résolu le problème de l'approximation de $Z$, puis traiter cette dernière question.

\section{Discrétisation de la méthode en $e$.}

2.1 ElÉMENTS FINIS. - Supposons construit sur D un pavage de tétraèdres, deux tétraèdres n'ayant en commun qu'une facette, une arête, un sommet, ou rien. (On s'arrange pour que les conducteurs soient eux-mêmes pavés par des sous-familles de tétraèdres.) Soit $\mathcal{N}$ l'ensemble des sommets (ou «nœuds ») du réseau ; $A$ l'ensemble des arêtes. Soit 
$\mathcal{N}_{0}$ l'ensemble des seuls sommets intérieurs à D. Pour chaque sommet $n$ de $\mathcal{N}$, on définit une fonction $w_{n}$, nulle sur tous les tétraèdres n'ayant pas $n$ pour un de leurs sommets, égale à un sur $n$, et affine continue sur les tétraèdres restants. (C'est la «fonction-chapeau », bien connue, de la méthode des éléments finis, associée au nœud $n$.) Pour chaque arête $a$ (définie par ses extrémités $n$ et $m$ ) on pose

$$
w_{a}=w_{n} \operatorname{grad} w_{m}-w_{m} \operatorname{grad} w_{n} .
$$

(Autre notation possible : $w_{\{n, m\}}$.) Le champ de vecteurs ainsi obtenu, nul sauf sur les tétraèdres qui ont l'arête $a$ en commun, est tel que sa partie tangentielle soit continue au passage des facettes du pavage. De ce fait, toute combinaison linéaire des $w_{a}$ à coefficients réels, telle que

$$
e=\sum_{a} \bar{e}_{a} w_{a} \quad(a \in \mathcal{A}),
$$

représente un champ de $\mathbb{E}(\mathrm{D})$, par construction. De même, toute combinaison

$$
\psi=\sum_{n} \bar{\psi}_{n} w_{n} \quad\left(n \in \mathcal{N}_{0}\right)
$$

où tous les $\psi_{n}$ ont la même valeur (le $c_{i}$ de (27)) sur chaque $C_{i}$, est une fonction de $\Psi^{0}(D)$. Noter que si $\psi$ est de la forme (23), $\bar{\psi}_{n}$ est la valeur de $\psi$ en $n$. De même, si $e$ est de la forme (22), $\bar{e}_{a}$ est la circulation de $e$ le long de l'arête $a$. Au lieu de (23), on peut écrire

$$
\psi=\sum_{n \in \mathcal{N}_{00}} \bar{\psi}_{n} w_{n}+\sum_{i=1, \ldots, m} c_{i} w_{i}
$$

où $w_{i}$ est la somme des $w_{n}$ pour tous les nœuds de $C_{i}, \mathcal{N}_{00}$ étant l'ensemble des nœuds intérieurs à $\mathrm{D}-(\mathrm{C}-\mathrm{G})$.

Remarque 4. Une généralisation utile est possible, dans le cas où les pavés ne seraient pas des tétraèdres droits mais des images continues de tétraèdres, à facettes non planes. Il suffit en effet que les fonctions $w_{n}$ vérifient

$$
\sum_{n} w_{n} \equiv 1 \quad(n \in \mathcal{N})
$$

pour que tout ce qu'on a dit (et tout ce qu'on va en déduire) reste valable. Cela tient en particulier à ce que la circulation de $w_{a}$ le long de $a$ est toujours égale à 1 (et à 0 le long des autres arêtes) si les $w_{n}$ ont cette propriété, à condition de prendre pour abscisse curviligne, le long de l'arête $a=\{n, m\}$, précisément $w_{m}$ (ou $1-w_{n}$, qui lui est égal) multiplié par la longueur de l'arête. (La remarque 4, pour évidente qu'elle soit, ne semble pas avoir été faite antérieurement.)
Remarque 5. On a

$$
\operatorname{grad} w_{n}=\sum_{m} w_{\{m, n\}} \quad(m \in \mathcal{N})
$$

comme conséquence immédiate de (24): donc l'espace engendré par les champs d'arêtes (21) contient les gradients des fonctions nodales. Cette propriété va jouer un rôle essentiel. Elle implique (et, à toutes fins utiles, remplace, en beaucoup plus simple) la fameuse "condition de Brezzi-Babuska » $[1,4]$ de la théorie des éléments mixtes.

L'idée de la méthode de Galerkine consiste à chercher $e$, solution de (20), sous la forme approchée

$$
e=\sum_{a} \bar{e}_{a} w_{a} \quad(a \in \mathcal{A})
$$

avec les circulations $\bar{e}_{a}$ (fonctions du temps) comme inconnues. Puisque $e$ n'est pas, sauf accident, de la forme (25), on ne peut espérer satisfaire (20) pour tous les «champs-tests » $e^{\prime}$ et toutes les «fonctionstest " $\psi^{\prime}$. Mais en se contentant des $e^{\prime}$ de la forme (22) et des $\psi^{\prime}$ de la forme (23), on obtient un système d'équations différentielles :

$$
\begin{array}{r}
\int_{\mathrm{D}} \partial_{t}\left(\sigma e+j^{\mathrm{g}}\right) \cdot w_{a^{\prime}}+\int_{\mathrm{D}} \frac{1}{\mu} \operatorname{rot} e \cdot \operatorname{rot} w_{a^{\prime}}+ \\
+\frac{1}{\mu_{0}} \int_{\mathrm{S}} Z e_{\mathrm{S}} \cdot w_{a^{\prime}}=0 \quad \forall a^{\prime} \in \mathcal{A}_{0},
\end{array}
$$

et de relations algébriques :

$$
\begin{array}{r}
\int e \cdot \operatorname{grad} w_{n^{\prime}}=0 \quad \forall n^{\prime} \in \mathcal{N}_{00}, \int_{\forall i \in[1, m],} e \cdot \operatorname{grad} w_{i}=0 \\
\forall i \in[27)
\end{array}
$$

qui déterminent $e$ (de forme (25)) avec d'autant plus de précision que le pavage est plus fin.

2.2 NATURE DU MODÈLE DISCRET. - Vérifions que (26) est bien un système d'équations différentielles. Définissons les éléments de matrice suivants :

$$
\begin{aligned}
& \bar{A}_{a a^{\prime}}=\int_{\mathrm{D}} \sigma w_{a} \cdot w_{a^{\prime}} \quad\left(a \text { et } a^{\prime} \text { parcourant } \mathcal{A}\right), \\
& \bar{B}_{a a^{\prime}}=\int_{\mathrm{D}} \frac{1}{\mu} \operatorname{rot} w_{a} \cdot \operatorname{rot} w_{a^{\prime}} \quad(\text { id. }), \\
& \bar{Z}_{a a^{\prime}}=\int_{\mathrm{S}} Z\left(w_{a}\right)_{\mathrm{S}} \cdot w_{a^{\prime}} \quad(\text { id. }), \\
& \bar{D}_{a n^{\prime}}=\int_{\mathrm{D}} w_{a} \cdot \operatorname{grad} w_{n^{\prime}} \\
& \left(a \text { et } n^{\prime} \text { parcourant } \mathcal{A} \text { et } \mathcal{N}_{00}\right),
\end{aligned}
$$

et les composantes de vecteur suivantes :

$$
\bar{j}_{a^{\prime}}=\int_{\mathrm{D}} j^{\mathrm{g}} \cdot w_{a^{\prime}} \quad\left(a^{\prime} \text { parcourant } \mathfrak{A}\right) .
$$


Si l'on note $\bar{e}$ le vecteur des valeurs $\left\{\bar{e}_{a}: a \in \mathcal{A}\right\}$ (vecteur qui est fonction du temps), on voit que (26) et (27) s'écrivent

$$
\begin{gathered}
\partial_{t} \bar{A} \bar{e}+\left(\bar{B}+\frac{1}{\mu_{0}} \bar{Z}\right) \bar{e}=-\partial_{t} \bar{j} \\
\bar{D} \bar{e}=0
\end{gathered}
$$

donc un système différentiel et un système algébrique, comme annoncé.

Remarque 6. Si le calcul de $\bar{A}, \bar{B}$ et $\bar{D}$ - calcul qu'on appelle «assemblage » dans la méthode des éléments finis - ne pose aucun problème, il faudra par contre revenir sur celui de $\bar{Z}$. Noter que les seuls coefficients non nuls dans $\bar{Z}$ sont ceux correspondant aux arêtes contenues dans $\mathrm{S}$. Noter aussi que $\bar{A}, \bar{B}$ et $\bar{D}$ sont très creuses (peu de coefficients non nuls). Tout ceci rend la résolution de (28) (29) praticable, malgré le nombre d'inconnues (plusieurs dizaines de milliers, typiquement).

Comme $\bar{A}, \bar{B}, \bar{Z}$ sont des matrices carrées, et qu'il y a donc en apparence autant d'équations que d'inconnues dans (28), on peut se demander quelle est la raison d'être de (29). Elle réside dans le fait que $\bar{A}$ n'est pas régulière : comme $\sigma=0$ dans $\mathrm{D}-(\mathrm{C}-\mathrm{G}), \bar{A}_{a a^{\prime}}=0$ pour toute arête $a$ dans cette région. Donc les équations (28) dégénèrent, pour ces arêtes-là, en simples relations algébriques. Mais celles-ci non plus ne sont pas de rang maximal. En effet, l'équation (26) est automatiquement satisfaite pour tout $e$ de la forme

$$
\begin{aligned}
& e=\sum_{n} \bar{\psi}_{n} \operatorname{grad} w_{n}+c_{i} \operatorname{grad} w_{i} \\
& \qquad\left(n \in \mathcal{N}_{00}, i=1, \ldots, m\right)
\end{aligned}
$$

(puisque rot $e=0$ et $e=0$ là où $\sigma>0$ ). Or un tel champ est bien de la forme (25) (cf. la Remarque 5). Donc (26) ne détermine pas $e$ de façon unique. Le rôle de (29) est de rétablir cette unicité. (On le vérifie sans peine, en notant d'abord que le nombre d'équations dans (29) est bien celui des $\bar{\psi}_{n}$ et des $c_{i}$ de (30).)

Une autre façon de voir ce qui se passe est d'écrire un schéma de Crank-Nicolson pour (28). Chaque pas consiste à résoudre un système linéaire dont la matrice est la sous-matrice de $\bar{A}+\Delta t(\bar{B}+\bar{Z})$ correspondant aux arêtes de $\mathcal{A}_{0}$. Cette matrice n'est pas régulière, mais le devient si on se restreint aux $\bar{e}$ satisfaisant (29).

Remarque 7. Il y a une autre façon de traiter le problème de dégénérescence posé par le fait que $\sigma=0$ hors des conducteurs. On note que, dans $\mathrm{D}-(\mathrm{C}-\mathrm{G})$,

$$
\operatorname{rot}\left(\frac{1}{\mu} \operatorname{rot} e\right)=-\partial_{t} j^{\mathrm{g}}, \quad \operatorname{div} e=0 .
$$

Il existe donc (en supposant $D-(C-G)$ simplement connexe, mais cette restriction peut être levée) un potentiel $\varphi$ (le potentiel magnétique, à une dérivation près en temps) et un certain champ $k^{g}$, calculable, tels que

$$
\operatorname{rot} e=\mu \operatorname{grad} \varphi+k^{g} .
$$

Il est naturel de représenter $\varphi$ par ses valeurs nodales. Alors l'équation (31) devient, sous forme variationnelle discrète,

$$
\int_{\mathrm{D}-\mathrm{C}} \mu \operatorname{grad} \varphi \cdot \operatorname{grad} \varphi^{\prime}=\sum_{i} \int_{\partial \mathrm{C}_{i}}\left(g-n \cdot k^{g}\right) \varphi^{\prime}
$$

où $g$ est la composante normale $n$. rot $e$, que l'on peut obtenir à partir des valeurs d'arête de $e$. On voit s'esquisser ainsi une méthode où les inconnues seraient les circulations de $e$ le long des arêtes conductrices et les valeurs nodales de $\varphi$ dans l'air. C'est la « méthode $a-\varphi$ » employée par plusieurs auteurs [9], à cela près qu'ils utilisent des éléments vectoriels nodaux pour $e$ au lieu d'éléments d'arêtes. (L'avantage des éléments d'arête se fait sentir lorsque la conductivité $\sigma$ n'est pas partout la même ; cf. [8].)

2.3 CoMment CALCuler $\bar{Z}$ ? - Soit $\mathfrak{A}_{\mathrm{S}}$ l'ensemble des arêtes appartenant à $S$. Le problème est de trouver une approximation de $Z$ pour $u_{\mathrm{S}}$ de la forme

$$
u_{\mathrm{S}}=\sum_{a} \bar{u}_{a} w_{a} \quad\left(a \in \mathcal{A}_{\mathrm{S}}\right)
$$

où $w_{a}$ dénote cette fois la trace tangentielle du champ d'arête défini en (21).

La méthode suivie est classique en théorie du potentiel, mais quelques rappels sont nécessaires. Soit $j$ un champ surfacique, défini sur $\mathrm{S}$, vérifiant

$$
\operatorname{div}_{S} j=0 \text {. }
$$

(On définit la divergence surfacique $\operatorname{div}_{\mathrm{S}}$ à l'aide du théorème de Stokes, tout comme en dimension 3.) Posons, dans tout l'espace,

$$
u(x)=\frac{1}{4 \pi} \int_{\mathrm{S}} \frac{j(y)}{|x-y|} \mathrm{d} y \text {. }
$$

Il est bien connu que $\operatorname{div} u=0$ (grâce à (33)) et que rot rot $u=0$ dans $\mathrm{E}-\mathrm{S}$, donc $u$ satisfait bien (16). Pour tenter d'obtenir $n \wedge$ rot $u$, imaginons le champ de normales sur $S$ prolongé, dans un voisinage de $S$, en un champ régulier, que l'on note encore $n$. Alors, si $x$ n'est pas dans $\mathrm{S}$, on a

$$
\operatorname{rot} u=x \rightarrow \frac{1}{4 \pi} \int_{\mathrm{S}} \frac{(x-y) \wedge j(y)}{|x-y|^{3}} \mathrm{~d} y,
$$


d'où le champ $n \wedge$ rot $u$ hors de $\mathrm{S}$ :

$n \wedge \operatorname{rot} u=x \rightarrow \frac{1}{4 \pi} \int_{\mathrm{S}} n(x) \wedge \frac{(x-y) \wedge j(y)}{|x-y|^{3}} \mathrm{~d} y$.

Supposons que $x$ suive une ligne de champ du champ $n$ (Fig. 3), soit $\gamma$, coupant $S$ au point $\xi$. Nous sommes intéressés à la limite «de l'extérieur» de $n \wedge \operatorname{rot} u$ au point $\xi$, soit $(n \wedge \operatorname{rot} u)_{\mathrm{e}}$. On démontre que le saut de $n \wedge$ rot $u$, soit

$$
[n \wedge \operatorname{rot} u]=(n \wedge \operatorname{rot} u)_{\mathrm{e}}-(n \wedge \operatorname{rot} u)_{\mathrm{i}},
$$

c'est-à-dire la différence entre les limites « de l'extérieur » et « de l'intérieur», est égal à $j$ (ce que l'on comprendra immédiatement en pensant à $n \wedge$ rot $u$ comme au champ magnétique dû au courant $j$, et en appliquant la règle d'Ampère).

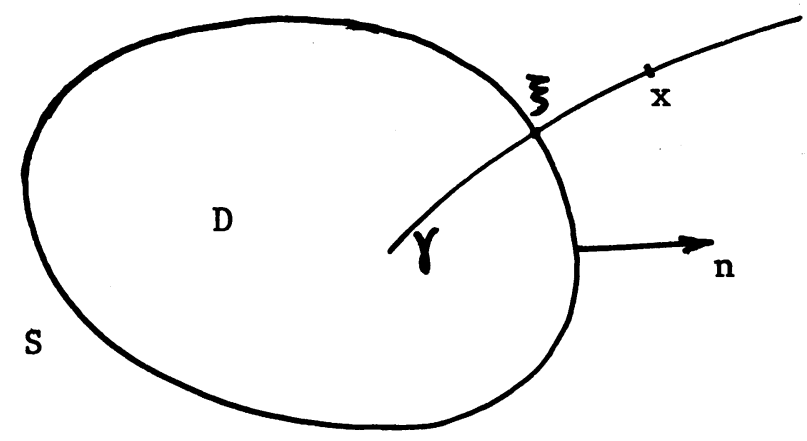

Fig. 3. - Notations relatives au calcul de $Z$.

[Notation, as regards the determination of $Z$.]

Or, la quantité à droite de (35) est parfaitement définie même si $x$ fait partie de $S$ (pour peu que $S$ soit assez régulière), puisque l'intégrale converge pour tout champ $j$ régulier. Notons $H^{\mathrm{t}} j$ le champ surfacique ainsi obtenu (on verra plus loin la raison d'être du t). L'étude détaillée de (35) lorsque $x$ tend vers $\xi$ montrerait que

$$
H^{\mathrm{t}} h(\xi)=\left((n \wedge \operatorname{rot} u)_{\mathrm{e}}+(n \wedge \operatorname{rot} u)_{\mathrm{i}}\right) / 2 .
$$

Par conséquent,

$$
\begin{aligned}
Z u_{\mathrm{S}} \equiv(n \wedge \operatorname{rot} u)_{\mathrm{e}} & =\frac{1}{2}[n \wedge \operatorname{rot} u]+H^{\mathrm{t}} j \\
& =\left(1 / 2+H^{\mathrm{t}}\right) j .
\end{aligned}
$$

Reste à éliminer $j$ entre (34) et (36). Notons $G$ l'opérateur intégral de (36), en se restreignant aux $x$ de $\mathrm{S}$, de sorte que $u_{\mathrm{S}}=G j$. Nous voyons alors que

$$
Z=\left(1 / 2+H^{\mathrm{t}}\right) G^{-1} \text {. }
$$

Comme l'opérateur $Z$ est auto-adjoint, tout comme $G$, nous avons aussi $Z=G^{-1}(1 / 2+H)$ (où l'on voit le sens du $t$ : transposition de $H$ ), et donc

$$
1 / 2+H=G Z,
$$

c'est-à-dire, sous forme développée (cf. Remarque 8 pour le détail du calcul), ceci :

$$
\begin{array}{r}
\frac{1}{2} u_{\mathrm{S}}(x)+\frac{1}{4 \pi} \int_{\mathrm{S}} \mathrm{d} y \frac{x-y}{|x-y|^{3}} \wedge\left(n(y) \wedge u_{\mathrm{S}}(y)\right)= \\
=\frac{1}{4 \pi} \int_{\mathrm{S}} \frac{\mathrm{d} y}{|x-y|}\left(Z u_{\mathrm{S}}\right)(y)
\end{array}
$$

qui est analogue à la « troisième formule de Green » utilisée en méthodes intégrales, et en particulier dans la méthode h-phi (voir [3], formule (30)).

Nous utiliserons (38) plutôt sous forme variationnelle :

$$
\begin{aligned}
\frac{1}{2}\left(u_{\mathrm{S}}, j^{\prime}\right)+\left(H u_{\mathrm{S}}, j^{\prime}\right) & =\left(G Z u_{\mathrm{S}}, j^{\prime}\right) \quad \forall j^{\prime} \in J \\
& =\left(Z u_{\mathrm{S}}, G j^{\prime}\right) \quad \forall j^{\prime} \in J
\end{aligned}
$$

où $J$ est l'espace des champs tangentiels réguliers, et où $(, \quad)$ est le produit scalaire de deux champs tangentiels, c'est-à-dire

$$
\left(u, u^{\prime}\right)=\int_{\mathrm{S}} u(x) \cdot u^{\prime}(x) .
$$

Remarque 8. Pour être complet, nous donnons cidessous le calcul de l'expression de $H$ qui a été utilisée dans (38), à partir de celle de $H^{\mathrm{t}}$. Si $u$ est un champ tangentiel,

$$
\begin{aligned}
& (H u, j)=\left(u, H^{\mathrm{t}} j\right) \\
& =\frac{1}{4 \pi} \int_{\mathrm{S}} \mathrm{d} x u(x) \cdot \int_{\mathrm{S}} \mathrm{d} y n(x) \wedge \frac{(x-y) \wedge j(y)}{|x-y|^{3}} \\
& =\frac{1}{4 \pi} \iint_{\mathrm{S}} \mathrm{d} x \mathrm{~d} y\left(u(x), n(x), \frac{(x-y) \wedge j(y)}{|x-y|^{3}}\right) \\
& =\frac{1}{4 \pi} \iint_{\mathrm{S}} \mathrm{d} y j(y) \cdot \int_{\mathrm{S}} \mathrm{d} x(u(x) \wedge n(x)) \wedge \frac{x-y}{|x-y|^{3}}
\end{aligned}
$$

d'où (en permutant $x$ et $y$ )

$$
H u=x \rightarrow \frac{1}{4 \pi} \int_{\mathrm{s}} \mathrm{d} y \frac{x-y}{|x-y|^{3}} \wedge(n(y) \wedge u(y)) .
$$

Maintenant, revenons à l'objet du calcul. Il s'agit d'évaluer $\left(Z u_{\mathrm{S}}, u_{\mathrm{S}}^{\prime}\right)$ lorsque $u_{\mathrm{S}}$ est de la forme (32) et que

$$
u_{\mathrm{S}}^{\prime}=\sum_{a} \bar{u}_{a}^{\prime} w_{a} \quad\left(a \in \mathcal{A}_{\mathrm{S}}\right)
$$

D'après (39), cela revient à évaluer le premier membre de (39) pour $j^{\prime}$ tel que $G j^{\prime}=u_{\mathrm{S}}^{\prime}$. La difficulté, bien sûr, est qu'on ne sait pas trouver un tel $j^{\prime}$ autrement que de façon approchée.

Soit donc $j_{k}, k=1, \ldots, p$, une base de courants surfaciques sur $\mathrm{S}$, vérifiant $\operatorname{div}_{\mathrm{S}} j_{k}=0$. (On verra plus loin comment la construire.) On va chercher des coefficients $\bar{q}_{k}$ tels que si $j^{\prime}=\Sigma_{k} \bar{q}_{k} j_{k}$, alors $G j^{\prime}=u_{\mathrm{S}}^{\prime}$ soit approximativement réalisé. 
Pour cela, définissons les éléments de matrices suivants :

$$
\begin{array}{r}
\bar{G}_{k l}=\frac{1}{4 \pi} \iint_{\mathrm{S}} \mathrm{d} x \mathrm{~d} y \frac{j_{k}(x) \cdot j_{l}(y)}{|x-y|} \quad(k, l=1, \ldots, p) \\
\bar{B}_{a k}=\int_{\mathrm{S}} \mathrm{d} x w_{a}(x) \cdot j_{k}(x) \quad\left(a \in \mathcal{A}_{\mathrm{S}}, k=1, \ldots, p\right)
\end{array}
$$

et posons ( $\bar{q}$ est le vecteur des $\bar{q}_{k}$ et $\bar{u}_{\mathrm{S}}^{\prime}$ celui des $\bar{u}_{\mathrm{a}}^{\prime}$ de (41)) :

$$
\bar{q}=\bar{G}^{-1} \bar{B} \bar{u}_{\mathrm{S}}^{\prime}
$$

Cela donne la meilleure approximation de $j^{\prime}$ au sens de la norme $(G j, j)^{1 / 2}$. Si maintenant

$$
\begin{aligned}
& \bar{H}_{a k}= \\
& =\frac{1}{4 \pi} \iint_{\mathrm{S}} \mathrm{d} x \mathrm{~d} y\left(\frac{x-y}{|x-y|^{3}}, n(y) \wedge w_{a}(y), j_{k}(x)\right),
\end{aligned}
$$

(39) devient l'approximation

$$
\left((\bar{B} / 2+\bar{H}) \bar{u}_{\mathrm{S}}, \bar{G}^{-1} \bar{B} \bar{u}_{\mathrm{S}}^{\prime}\right) \simeq\left(\bar{Z} \bar{u}_{\mathrm{S}}, \bar{u}_{\mathrm{S}}^{\prime}\right)
$$

où $\bar{u}_{\mathrm{S}}$ est le vecteur des $\bar{u}_{a}$ de (32). Comme le second membre est une forme bilinéaire symétrique, le premier l'est aussi, d'où l'approximation

$$
\bar{Z} \simeq \operatorname{sym}\left(\bar{B}^{\mathfrak{t}} \bar{G}^{-1}(\bar{B} / 2+\bar{H})\right)
$$

(avec $t$ pour «transposée " et sym pour "partie symétrique »).

On vient donc de trouver l'approximation souhaitée de l'opérateur $Z$, sous la forme d'une matrice $\bar{Z}$ opérant sur le vecteur des degrés de liberté d'arêtes surfaciques. Le calcul de $\bar{Z}$ n'a rien de trivial, puisqu'il repose sur l'inversion de $\bar{G}$, une matrice symétrique pleine. Quant au calcul des éléments de $\bar{B}, \bar{H}$, et $\bar{G}$ elle-même, il est plus ou moins simple selon le choix des $j_{k}$. Le plus simple qu'on puisse rechercher est $j_{k}$ constant par triangles. Mais il faut aussi que $\operatorname{div}_{\mathrm{S}} j_{k}=0$.

Bien que ces deux exigences ne semblent guère compatibles, on peut les satisfaire en posant

$$
j_{k}=n \wedge \operatorname{grad} w_{k}
$$

où $k$ parcourt $\mathcal{N}_{\mathrm{S}}$, l'ensemble des nouds surfaciques.

\section{Conclusion.}

Il est donc possible, en combinant l'emploi d'éléments finis dans une partie de l'espace, incluant toute la région intéressante, et d'éléments de frontière pour tenir compte du comportement du champ dans le reste de l'espace, de calculer le champ électrique dans les conducteurs et autour d'eux dans le cas du problème pris ici pour modèle (et bien sûr dans toute configuration analogue).

Ce ne sont pas n'importe quels éléments finis, mais des éléments dits "mixtes", aux degrés de liberté associés aux arêtes du maillage et non aux nœuds. Les champs construits à partir de ces éléments ont les bonnes propriétés de continuité requises par la physique: continuité tangentielle de $e$, mais pas continuité normale. En fait, il est essentiel que la méthode numérique n'impose pas artificiellement de continuité normale au champ $e$, puisqu'on s'intéresse surtout à sa partie normale sur les surfaces conductrices, là où précisément une discontinuité normale apparaît.

La méthode intégrale elle-même présente des aspects nouveaux par rapport à d'autres applications du même concept $[3,8]$. Au lieu de passer par l'intermédiaire d'une couche de charges surfaciques, comme dans la méthode h-phi, on a fait appel ici à des courants superficiels.

Mais à part dela, les méthodes en $h$ et en $e$ sont très voisines : Ren et Bouillault [8] ont pu réaliser rapidement une implémentation de la méthode en $e$ à partir du code " Trifou », qui utilise $h$-phi [3]. Disposer des deux méthodes est de toute façon une bonne chose, à cause de leur complémentarité.

Un aspect de cette complémentarité est ce qu'on appelle la «conformité » des champs approchés obtenus. Dans la méthode en $e$, le champ électrique a la continuité tangentielle requise (on dit qu'il est « rot-conforme »), par construction, et donc $b$ obtenu par $\partial_{t} b=-$ rot $e$, est «div-conforme » (continuité normale). Mais $h$ n'est pas rot-conforme, $j$ n'est pas div-conforme. Cette dernière caractéristique est d'ailleurs le premier inconvénient de la méthode en $e$ : la relation $n \cdot j=0$ sur les surfaces conductrices n'est vérifiée qu'au sens faible, donc en moyenne, et on peut donc sur les visualisations du courant voir les lignes de courant sortir du conducteur à certains endroits. La méthode en $h$, qui assure rot-conformité de $h$ et donc div-conformité de $j$, n'a pas ce défaut-là, mais a le défaut complémentaire en ce qui concerne $b$ : le flux de $b$ n'est conservé qu'en moyenne, au sens faible. Donc si on tient à cette conservation, c'est-à-dire à la conformité de $b$, il faut utiliser la méthode en $e$.

Enfin, la méthode en $e$ a l'avantage de fournir le champ électrique hors des conducteurs. (On pourrait l'obtenir avec la méthode $h$-phi, mais pas directement, seulement par un calcul complémentaire.) Mais il faut bien prendre garde à la modélisation du générateur à cet égard. Le cas traité ici (pas de charges dans G) est un cas idéal. Dans un prochain travail, nous verrons comment adapter la méthode générale présentée ici à des situations particulières. 


\section{Bibliographie}

[1] BABUSKA I., Error bounds in the finite element method, Numer. Math. 16 (1971) 322-33.

[2] Bossavit A., Two dual formulations of the 3-D eddycurrents problem, COMPEL 4 (1984) 103-16.

[3] Bossavit A., Le calcul des courants de Foucault, en trois dimensions, en présence de corps à haute perméabilité magnétique, Revue Phys. Appl. 23 (1988) 1147-59.

[4] BREZZI F., On the existence, uniqueness and applications of saddle-point problems arising from Lagrangian multipliers, RAIRO Anal. Numer. R 2 (1974) 129-51.

[5] CARPenter C. J., Comparison of alternative formulations of 3-dimensional magnetic-field and eddycurrent problems at power frequencies, Proc. IEE 124 (1977) 1026-34.
[6] Chaussecourte P., Bossavit A., Vérité J. C., Crutzen Y. R., 3D Eddy Current Distribution in a Tokamak First Wall During a Plasma Disruption Using "Trifou », Int. Symp. on Fusion Nuclear Technology (1988) Tokyo.

[7] EMSON C. R. I., SimKIN J., An optimal method for 3D eddy-currents, IEEE Trans. MAG-19 (1983) 2450-52.

[8] Ren Z., Bouillault F., Razek A., Bossavit A., VÉrité J. C., A New Hybrid Model Using Electric Field Formulation for 3-D Eddy Current Problems, in Proc. Conf. COMPUMAG, Tokyo, 1989, à paraître, IEEE Trans. Magn. (1990).

[9] Trowbridge C. W., Low Frequency Electromagnetic Field Computation in Three Dimensions, Comp. Meth. Appl. Mech. Eng. 52 (1985) 653-74. 